\title{
Vulnérabilités et recompositions baltiques : les conséquences d'une géopolitique insidieuse
}

Baltic vulnerability and recomposition: the consequences of an insidious geopolitics

Vulnerabilidades y recomposiciones bálticas: las consecuencias de una geopolítica insidiosa

\section{Nicolas Escach}

\section{(2) OpenEdition}

Journals

Édition électronique

URL : https://journals.openedition.org/mappemonde/5426

DOI : $10.4000 /$ mappemonde.5426

ISSN : 1769-7298

Éditeur

UMR ESPACE

\section{Référence électronique}

Nicolas Escach, « Vulnérabilités et recompositions baltiques : les conséquences d'une géopolitique insidieuse ", Mappemonde [En ligne], 130 | 2021, mis en ligne le 15 mars 2021, consulté le 20 mai 2021. URL : http://journals.openedition.org/mappemonde/5426 ; DOI : https://doi.org/10.4000/ mappemonde. 5426

Ce document a été généré automatiquement le 20 mai 2021.

\section{(c) (i)(2)}

La revue Mappemonde est mise à disposition selon les termes de la Licence Creative Commons Attribution - Pas d'Utilisation Commerciale - Partage dans les Mêmes Conditions 4.0 International. 


\title{
Vulnérabilités et recompositions baltiques : les conséquences d'une géopolitique insidieuse
}

\author{
Baltic vulnerability and recomposition: the consequences of an insidious \\ geopolitics \\ Vulnerabilidades y recomposiciones bálticas: las consecuencias de una \\ geopolítica insidiosa
}

Nicolas Escach

\section{Introduction}

1 Rarement, depuis le début de la décennie 1990, l'impact de la géopolitique sur la géographie des coopérations politiques n'a été aussi forte autour de la mer Baltique. Alors que la zone semblait élevée au rang de laboratoire de la gouvernance transnationale, une escalade d'intimidations entre l'OTAN, l'Union européenne, les États-Unis, la Russie et la Chine y a pris racine avec la crise ukrainienne de 2014. Une interrogation persiste d'ailleurs sur les termes à employer : tensions, rivalités, conflits, guerres ? L'adjectif « hybride » semble s'être en tout cas imposé, l'agitation protéiforme frôlant les lignes plus qu'elle ne les franchit (Murphy et al., 2016 ; Radin, 2017).

2 En mer Baltique, le choc a donc pris la forme d'une succession de secousses dont certaines sont restées peu visibles. La Baltique est l'espace des interactions à bas bruit, mais elle est aussi celui des dislocations légères, des conflits larvés, des intimidations sourdes, des altercations discrètes (bien que de plus en plus bruyantes médiatiquement). Une forte part dans ces dynamiques est laissée à la rumeur, à l'impalpable, parfois aux fake news. Comme pour les liens faibles, les brisures légères n'ont pas de force isolément, mais deviennent structurantes en raison de leur accumulation. Elles sont alors susceptibles de recomposer durablement les territoires nordico-baltiques à toutes les échelles. 
3 Le retour à une conflictualité larvée de la géographie régionale a influencé les publications scientifiques sur les espaces riverains. Les recherches sur la mer Baltique sont passées par trois périodes principales depuis la fin de la guerre froide, avec un net décalage de la France sur le monde universitaire allemand, scandinave, balte ou anglophone. Institutionnels et académiques se sont d'abord entendus, lors d'une première phase, autour d'une esquisse fantasmée de la région ${ }^{1}$. L'enjeu était de réconcilier des rives, trop longtemps divisées, par une coopération réticulaire jugée rédemptrice. La région baltique a cependant été très rapidement analysée sous un angle déconstructionniste, sa construction étant perçue avant tout comme un acte performatif (et comme le résultat d'une prophétie autoréalisatrice) ${ }^{2}$. Depuis le milieu des années $2000^{3}$, enfin, les études baltiques abordent non seulement les réseaux d'échanges et de transferts (Arnaud Serry, Katerina Kesa), mais aussi les nombreux conflits qui la traversent. La Baltique a de nouveau fait son entrée dans la géopolitique en tant que nœud géostratégique d'importance mondiale (Chillaud, 2007 ; Bayou, 2015 ; Escach, 2016).

4 Alors que dans les années 1990, la fin d'un siècle marqué par une succession de conflits mondiaux (Seconde Guerre mondiale, guerre froide) conduisait à recentrer l'attention sur l'échelle régionale, le lac baltique s'effrite aujourd'hui à mesure qu'il est recontextualisé à une échelle locale, européenne, eurasiatique, voire mondiale. Des thématiques moins idylliques naissent de ce décentrement : les migrations et la montée de l'extrême droite, la fermeture des frontières et les conséquences sur les ensembles transfrontaliers, les rivalités locales et régionales autour de l'énergie, les conflits d'usage provoqués par la planification maritime, les accrochages militaires et diplomatiques entre Union européenne, OTAN et Russie. D'autres thèmes comme le tourisme (Lauriane Letocart) ou le développement durable (Teva Meyer, Grégoire Tortosa) apparaissent, suivant ainsi des tendances structurantes de la recherche et de la pratique (interrogations des acteurs riverains depuis le début de la décennie 2000 sur l'attractivité de la zone baltique, débats nationaux et régionaux autour de la définition des mix énergétiques et des sources d'énergie, innovations urbaines liées au traitement de la pollution...). La Baltique sort des quelques fils directeurs dans lesquels elle avait été emprisonnée: l'impasse de l'identité/des identités ${ }^{4}$ (en sciences politiques), l'aveuglement par la régionalisation et, parfois même, par la réticularité (en géographie), le piège déterministe (en sciences naturelles).

Dans cet article, nous centrerons la réflexion autour d'un terme plus souvent associé à un équilibre environnemental qu'à un équilibre géopolitique : la "vulnérabilité » de l'espace baltique. Nous choisirons de la définir davantage en la rapprochant du champ lexical de la résilience ${ }^{5}$ qu'en nous référant aux travaux sur le risque. Dans un contexte de fortes perturbations, la vulnérabilité est la difficulté d'une organisation spatiale donnée à se protéger, à s'adapter et/ou à se réorganiser. Souvent cumulative, elle est la conséquence d'un choc difficile à amortir (par sa fulgurance, sa répétition, son étendue géographique), mais aussi la cause d'une moindre capacité à réagir face à des évolutions futures (difficile prévisibilité des blessures). Elle peut être définie littéralement, selon nous, comme la sensibilité à l'endommagement d'un système spatial donné dans l'ensemble de ses composantes et de ses enjeux, ici le système spatial baltique pourtant habituellement associé à une robustesse coopérative. L'interterritorialité active, se traduisant par un ensemble de réseaux transfrontaliers, transnationaux et interrégionaux, était déjà la conséquence d'une difficulté des acteurs 
locaux riverains à s'insérer directement dans les processus de mondialisation et d'européanisation (Escach, 2014). Avec le retour des tensions, elle est désormais contrariée de l'intérieur, la densité des liens fonctionnels et politiques se retournant contre les territoires lorsqu'ils sont fragilisés. La résilience de l'espace dans son ensemble est donc à interroger. Quelle géographie l'impact de la révélation des tensions en mer Baltique (et non de la montée des tensions, elles sont en réalité plus anciennes) dessine-t-il sur la trajectoire des territoires riverains à toutes les échelles ? Nous reviendrons tout d'abord sur le contexte conflictuel dans la zone baltique, puis sur les signaux faibles de fragilité laissés par les conflits hybrides régionaux, avant de questionner l'impact de vulnérabilités multiformes sur la recomposition et la résilience des territoires baltiques.

6 L'analyse sera accompagnée d'une démarche chorématique originale révélant la configuration spatiale des tensions baltiques (rarement abordées sous un prisme géographique), ainsi que d'un travail de cartographie subjective, afin de représenter systématiquement les confrontations géopolitiques sous l'angle des principaux acteurs impliqués. Les représentations graphiques ouvriront également de nouvelles perspectives d'orientation ou de projection, la zone baltique étant trop souvent perçue en France comme un angle mort de l'Union européenne. Au sens de l'Europe géographique (de l'Atlantique à l'Oural), voire d'un potentiel carrefour émergent entre Union européenne, Asie et Arctique, la Baltique apparaît au contraire, de plus en plus, comme un "point nodal en devenir " vers lequel convergent les routes terrestres, fluviales et maritimes le long de transects ouest/est et nord/sud. Ces parcours en diagonale marquent un retour aux grandes traversées isthmiques sur les traces de l'ancienne route des Varègues aux Grecs. Le recours à la carte aura donc plusieurs objectifs dans cet article : décentrer notre regard pour mieux appréhender la situation d'entre-deux des confins européens, percevoir les conséquences territoriales d'une géopolitique baltique insidieuse ${ }^{6}$ à travers une approche différenciée par États impliqués, traduire le sentiment de menace graphiquement (par exemple, la peur d'une « insularisation » de la part des États baltes) et proposer des cartes inédites à plusieurs échelles pour illustrer un contexte régional de crise (à l'instar de la localisation des propriétés de la société Airiston Helmi dans l'archipel de Turku, ou des liens fonctionnels entre la Baltique orientale et la Russie, lesquels marquent une persistance des territoires à côté du "retour à l'Europe »). La Baltique souffre de manière plus générale d'une "carence cartographique " (pas d'atlas synthétique en dehors des réalisations de Pascal Orcier et de Teva Meyer), alors même qu'elle se trouve dans un espace sur lequel "l'inquiétude géographique » atteint un niveau très élevé et est diversement ressentie.

\section{L'héritage encore actif de la guerre froide}

7 La mer Baltique a été historiquement davantage marquée par des périodes de conflit que par des cycles de coopération (localisés au XXe siècle, entre les deux guerres et après la chute du Rideau de fer). Les tensions de la guerre froide n'ont d'ailleurs pas été complètement soldées et résolues jusqu'à aujourd'hui : le dernier traité frontalier signé entre Estonie et Russie date de 2014 et n'est pas encore entré en vigueur. Le début de la décennie 1990 constitue une parenthèse relativement factice, tant les organes régionaux ont cherché à étouffer les sujets conflictuels, les évacuant au profit d'une 
régionalisation soft. Anaïs Marin écrivait en 2009, à propos du Conseil des États de la mer Baltique, « les questions de sécurité "dure” furent d'emblée bannies de l'agenda, au profit d'une coopération moins formelle sur des sujets consensuels tels que l'environnement, l'économie ou la culture ${ }^{7}$."

8 L'entrée de la Pologne et des États baltes dans l'OTAN (1999 et 2004), et dans l'UE (2004), ont été les premiers catalyseurs d'un retour des tensions avec la Russie, qui considérait cet élargissement comme une ligne rouge à ne pas franchir. La pression russe sur l'est de la Baltique s'est cependant accélérée au cours de l'année 2007, juste avant la crise économique (Chalvin, 2007). Citons, entre autres événements, les réactions de Moscou autour de l'affaire du soldat de bronze de Tallinn, les sanctions économiques prises par des administrations ou des entreprises russes (appels à boycotter les produits estoniens, fermeture pour les poids lourds du pont transfrontalier de Narva, réduction annoncée du trafic ferroviaire à destination de l'Estonie sous prétexte de travaux sur les voies) ou encore la cyberattaque sur les sites institutionnels estoniens par la technique du déni de service (possiblement initiée par les services russes).

9 La crise ukrainienne de 2014 a donc davantage été un déclencheur au milieu d'un terrain déjà bien miné. Après la Géorgie et l'Ukraine, journalistes, élus et habitants ont craint des velléités russes sur la mer Baltique. Un article publié dans le quotidien Postimees et traduit par Courrier international titrait en 2014 «Daugavpils, la petite Crimée balte ». Des « Crimées » jaillissaient soudainement en Latgale (Lettonie) ou dans la région de Narva (Estonie). Ces régions sont en effet peuplées de russophones, parfois majoritairement ( $95 \%$ des habitants de Narva pratiquent le russe comme première langue). Elles captent des médias russes qui peuvent être de véritables vecteurs de propagande en insistant sur l'érosion des valeurs traditionnelles (entre autres par la reconnaissance des droits LGBT), les discriminations que subissent les minorités, le manque de fiabilité des alliés américains et européens, une prétendue renaissance du fascisme et la fragilité des gouvernements baltes ${ }^{8}$.

10 Certes, l'article 5 du Traité de l'Atlantique Nord est, normalement, à même de protéger la Pologne et les États baltes, membres de l'OTAN, mais son énoncé n'est pas exempt d'ambiguiités. La nécessaire réaction de l'Organisation en cas d'attaque dirigée contre un membre n'est pas décrite dans le détail et le texte précise même que le collectif devra prendre «telle action qu'elle jugera nécessaire, y compris l'emploi de la force armée ", comme pour inclure cette possibilité parmi d'autres. En réalité, l'article 5 aurait surtout une chance d'être activé si la Russie s'attaquait directement à un soldat de l'OTAN, pas forcément si elle violait les frontières orientales de l'UE. Qui serait prêt, en cas de nécessaire riposte, à mourir pour Daugavpils ? Le raisonnement est à peu près similaire pour l'article 42.7 du Traité de l'Union européenne, moins connu, qui précise qu'au cas où un État membre serait l'objet d'une agression armée sur son territoire « les autres États membres lui doivent aide et assistance par tous les moyens en leur pouvoir, conformément à l'article 51 de la charte des Nations unies ».

11 Les acteurs régionaux sont en réalité totalement partagés sur la nature de la crise à venir. Les services de renseignement estoniens sont plutôt alarmistes, jugeant dans un rapport publié en 2019 que les forces armées russes se préparent à une possible guerre le long d'un front étendu' ${ }^{9}$, qui ferait d'autant plus de dégâts que les États baltes présentent un relief plat. La Russie serait, pour d'autres spécialistes, plutôt intéressée par l'Asie centrale et une coopération active avec la Chine, afin de faire basculer vers l'est le centre de gravité eurasiatique, et se désintéresserait d'interventions militaires 
risquées en Europe, le cas ukrainien faisant exception pour des raisons historiques et stratégiques. Michel Foucher a, quant à lui, insisté à plusieurs reprises sur une possible instrumentalisation et dramatisation des événements baltiques qui aurait visé, notamment, à donner un second souffle à l'OTAN ${ }^{10}$.

\section{Les conflits hybrides comme vecteurs de fragilité territoriale}

12 Les tensions hybrides en mer Baltique, bien qu'anciennes ${ }^{11}$, ont connu une médiatisation sans précédent depuis la crise ukrainienne de 2014. Les mots tensions ou conflits nous semblent préférables au terme guerre, désignant une lutte armée ou des actes conflictuels frontaux particulièrement violents. Seul l'espace numérique peut justifier l'emploi d'un tel registre en raison d'attaques agressives recensées ces deux dernières décennies (2007 ayant constitué un point d'orgue), lesquelles ont généré d'importants dommages. Tensions et conflits se distinguent par l'engagement tangible de l'une au moins des parties prenantes sur différents modes d'affrontement. Il révèle le passage d'un état de dysfonctionnement ou de difficulté de coordination et d'échange (la tension) à une situation d'opposition frontale (le conflit). L'adjectif hybride traduit quant à lui une difficulté à classifier de nouvelles conflictualités baltiques pour cinq raisons principales. Tout d'abord, les oppositions chahutent les lignes plus qu'elles ne les remettent en cause. Les affrontements peuvent être durs, mais ils visent à toujours rester en dessous de la ligne rouge, « sous le seuil ». L'hybride crée alors une disproportion croissante entre des événements isolés et la montée d'une angoisse liée à la fois à la crainte d'une escalade et à la prise de conscience du potentiel de nuisance d'actions a priori anodines qui finissent par devenir structurantes en raison de leur accumulation et de leur inscription dans la durée. Les acteurs impliqués peuvent être étatiques et non étatiques, ce qui crée un troisième facteur d'hybridité. Les moyens d'action utilisés associent de plus, dans une visée hautement stratégique, déploiements militaires, mais aussi ingérence dans les débats politiques nationaux, sanctions économiques, cyber-opérations, campagnes de désinformation et de déstabilisation. L'objectif est souvent davantage de s'immiscer dans les consciences, les constructions idéologiques, les marqueurs territoriaux, que de gagner du terrain physique. L'hybride traduit enfin un croisement entre une confrontation manichéenne, largement instrumentalisée, des valeurs et des modèles et une implication plus indirecte et multilatérale de pays recherchant une influence économique et culturelle (ici les États-Unis et la Chine). Les conflits hybrides peuvent donc être définis comme un ensemble protéiforme d'événements, plus ou moins conventionnels, dont l'accumulation sur un même théâtre génère sur une temporalité longue, sans remise en cause des frontières, une fragilisation voire une érosion des systèmes territoriaux à toutes les échelles. Fantasmes, représentations, instrumentalisations et blessures plus tangibles se croisent, sans qu'il soit toujours possible de les distinguer.

13 Les tensions et conflits hybrides baltiques ont pris ces dernières années au moins huit formes géographiques (voir figure 1). Chacune matérialise une fragilisation, voire une archipélisation, des territoires baltiques qui n'est pas sans rappeler le roman d'Emmanuel Ruben La ligne des glaces (2014). 
Réalisation de motifs spatiaux à partir de la configuration géographique des tensions et conflits hybrides en mer Baltique

Roger Brunet a inventé le mot " chorème », en s'inspirant de la linguistique structurale, pour désigner un principe élémentaire de structuration de l'espace qui se représente par un modèle graphique (Brunet, 1980 ; Brunet, 1986). La pertinence des chorèmes repose sur le postulat que l'espace géographique produit par les sociétés est un système dynamique, structuré par des lois, et qu'il faut en découvrir la logique, la grammaire et l'organisation. La traduction spatiale des tensions et conflits hybrides en mer Baltique sous une forme chorématique a été réalisée grâce à un ensemble documenté de cas qui révèlent une opposition tangible ou symbolique. Ils ont été identifiés grâce à une veille de la presse nationale et régionale et grâce à un travail minutieux de terrain, puis de cartographie (à toutes les échelles). Les motifs spatiaux ainsi collectés offrent une vision géographique et plurielle des antagonismes baltiques tout en considérant l'espace comme un enjeu, voire un vecteur, des clivages régionaux.

Figure 1. Les motifs spatiaux des conflits hybrides

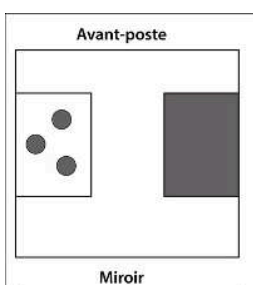

Miroir
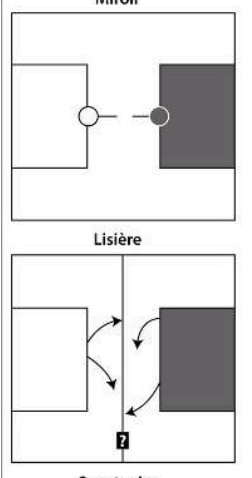

Sanctuaire

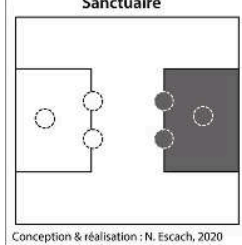

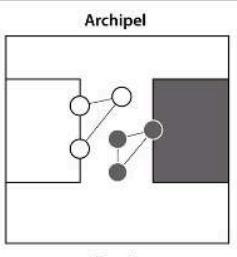

Front
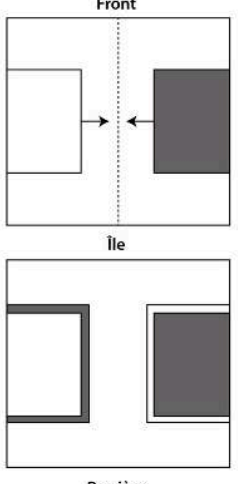

Barrière

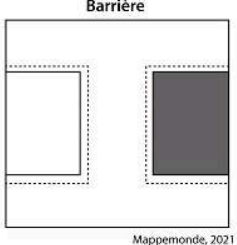

La recherche de l'avant-poste territorial ou du saut de puce est particulièrement visible à travers la déstabilisation des russophones en Estonie et en Lettonie et des implantations situées au cœur même des États nordiques et baltes qui, sous couvert d'activités classiques (tourisme, immobilier), sont soupçonnées de masquer une surveillance géostratégique. Elles participent d'une recherche de la projection avancée. En septembre 2018, l'archipel de Turku, situé près des îles Åland, à l'entrée du golfe de Botnie, a subi une importante perquisition menée par 400 membres des forces spéciales et garde-côtes finlandais ${ }^{12}$. 17 terrains et propriétés de la société immobilière à 
vocation touristique Airiston Helmi ont été fouillés, ce qui a abouti à la découverte de 3 millions d'euros en liquide, de coupures en diverses devises et de documents en langue étrangère. Le propriétaire, Pavel Melnikov, est un citoyen russe qui possède une dizaine d'îles entre l'île de Björkö et la ville de Pargas (notamment l'île de Säkkiluoto). Selon le service national cartographique de Finlande ${ }^{13}$, l'entreprise et l'homme d'affaires russe ont acheté des terrains et des bâtiments représentés sur la figure 2 pour un montant total d'environ 3,8 millions d'euros entre 2007 et 2014. L'homme est accusé par les autorités finlandaises de s'appuyer sur ces propriétés, minutieusement aménagées, pour bien d'autres finalités que le seul blanchiment d'argent. La zone pourrait être utilisée comme cheval de Troie dans des pays non membres de l'OTAN, donc jugés plus fragiles : choix des îles à proximité des bases militaires finlandaises ou des câbles sousmarins, pontons et débarcadères susceptibles d'accueillir une flotte militaire, présence d'héliports et de hangars pour hélicoptère, caméras de surveillance et cloisonnement, antennes paraboliques, habitations capables de loger plusieurs dizaines de personnes, plateformes en béton solide, centre de communication en sous-sol équipé de technologies de pointe. Tatu Mikkola, secrétaire général du Comité inter-administratif finlandais de sécurité, avait déclaré dès 2015 : «Les nouveaux phénomènes de guerre hybride sont les flux de réfugiés, les guerres de l'information et l'acquisition de terres à proximité de sites stratégiques ${ }^{14} »$. Le cas de Pavel Melnikov ne semble donc pas isolé. Depuis le début des années 2000, des particuliers et des entreprises russes ont acquis des terres, des propriétés, des hôtels et des magasins en Finlande, à proximité de zones d'entraînement, de garnisons, d'aéroports militaires, de couloirs de câbles de télécommunication, de réseaux électroniques, de jonctions ferroviaires ou de centrales nucléaires. Les littoraux du golfe de Botnie, et surtout la frontière orientale, concentrent la majeure partie des implantations (Hasanen, 2017) qui affichent pour la plupart des visées touristiques. Le long de la route nationale 6 , des magasins ouverts dans d'anciennes stations frontalières abandonnées, des hôtels, des stations-service, écoles ou centres de vacances seraient concernés. Au cours de l'été 2015, l'État finlandais est intervenu, pour la première fois, en exerçant une préemption foncière pour éviter l'achat par une société russe d'un important terrain de 80 hectares à proximité d'une zone militaire de tir sur la municipalité de Taipalsaari. Une note lui a été présentée le même été par le Comité de sécurité au sujet de la possibilité d'inscrire légalement la préemption foncière en cas de transactions douteuses près de sites stratégiques. Côté suédois, Jan Leijonhielm, directeur des études russes au sein de l'Agence suédoise de recherche pour la défense (FOI) a appelé, de son côté, à un renforcement législatif pour éviter l'achat par des citoyens russes de terrains à proximité de points de télécommunication stratégiques ou de zones militaires. La Russie semble donc, d'une manière plus insidieuse, se constituer elle aussi un «collier de perles » en Baltique. L'attention portée à l'enclave/exclave de Kaliningrad, très bien documentée par Frank Tétart (Tétart, 2007), participe du même phénomène d'avantposte. 
Figure 2. La politique foncière de la société Airiston Helmi dans l'archipel de Turku

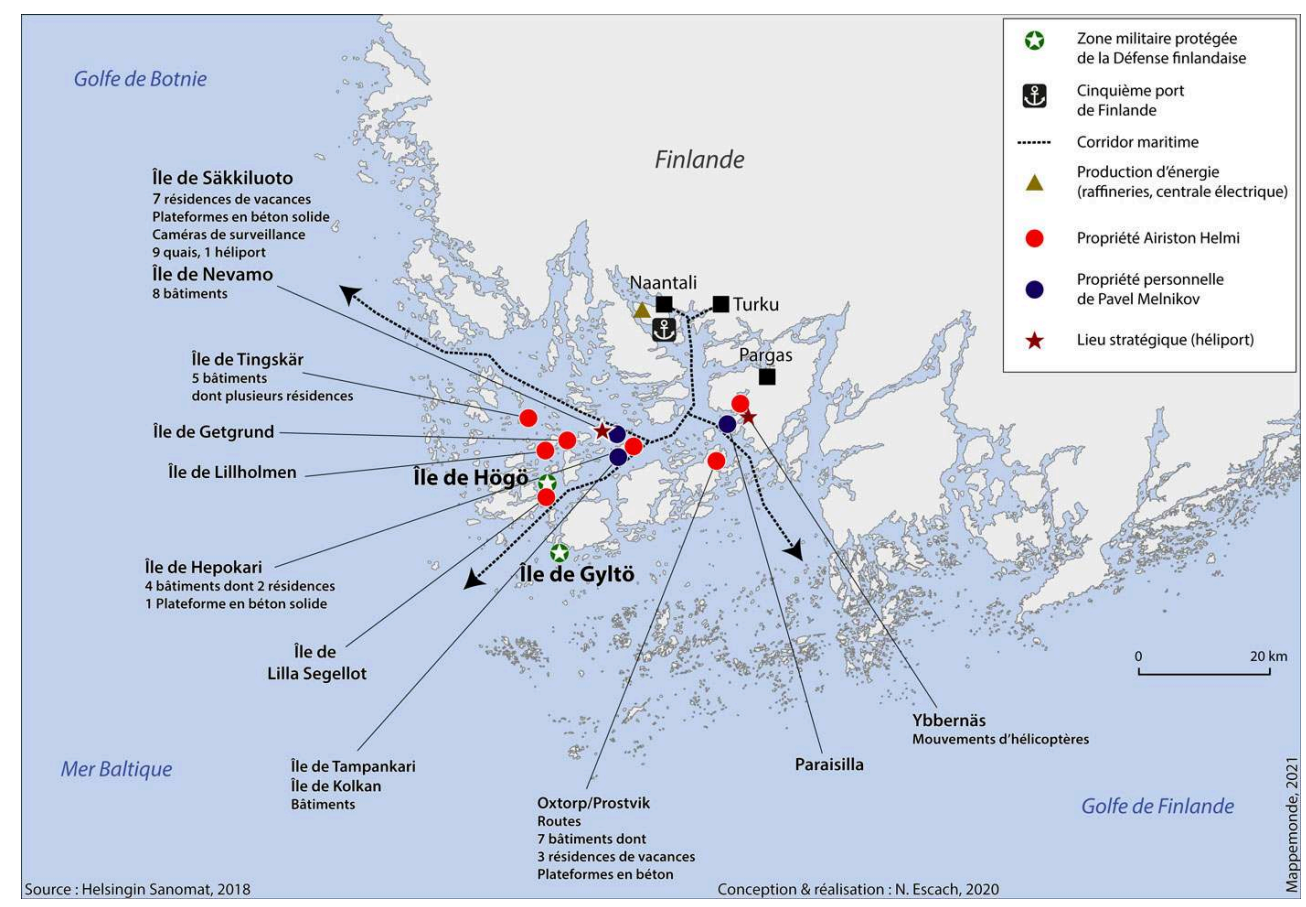

Le déploiement en réseau ou en archipel constitue une deuxième méthode. Russie, Union européenne et OTAN cherchent à s'approprier les flux et leurs supports techniques. Les conflits lors du lancement du gazoduc Nord Stream 1 se sont autant appuyés sur des questions économiques et écologiques que sur des enjeux géopolitiques (Larsson, 2007 ; Belligoli, 2012). Le contrôle des réseaux numériques est également un véritable enjeu, avec de fortes suspicions, côtés baltes et nordiques, sur l'existence d'une "usine à trolls » à Saint-Pétersbourg. La cyberattaque massive lancée contre l'Estonie en 2007 a des chances raisonnables d'avoir été commanditée depuis la Russie. Plus récemment, le renseignement norvégien a accusé la Russie d'avoir brouillé les signaux GPS lors d'exercices militaires et d'avoir équipé un béluga d'outils d'espionnage. La série Le Bureau des légendes a d'ailleurs mis en avant la guerre numérique menée par des centres d'excellence russes.

Les configurations de miroirs territoriaux sont également très nombreuses. Les exercices militaires sont un bon exemple d'intimidation mutuelle. Les États nordiques et baltes accusent notamment les Russes de ne pas suivre les procédures d'encadrement et de contrôle prévus dans le cadre de l'OSCE. Ils craignent un débordement, la plupart des interventions militaires russes ces dernières années ayant débuté par des exercices. Ceux-ci font largement appel à des acteurs extérieurs, comme en témoigne l'opération Joint Sea 2017 menée conjointement par la Russie et la Chine. Les scénarios fictifs établis pour l'entraînement des troupes sont eux-mêmes caractérisés par une figure spéculaire mettant en évidence une division simpliste et manichéenne entre forces du bien et forces du mal, laquelle apparait également sur certaines cartes russes (par exemple: https://eurasiaprospective.net/2017/09/18/la-baltique-enjeu-des-rivalites-otanrussiebret-sur-atlantico/). L'Arctic Challenge Exercise 2015, organisé par l'OTAN, a ainsi fait émerger deux États fictifs appelés Middleton et Woodland: « Nous nous trouvons dans le Middleton. Dans le nord, il y a un autre pays appelé Woodland. La frontière entre Middleton et Woodland va d'est en ouest, et se trouve à environ 1600 kilomètres au sud 
de l'autoroute 97. Dans la région, il existe depuis quelque temps un vieux conflit frontalier qui vient de resurgir ${ }^{15}$. »

La vieille organisation le long d'un front n'est pas absente du conflit hybride entre Russie, pays européens et OTAN. La sécurisation des frontières orientales de l'Union européenne (construction de barrière et de clôtures, militarisation) démontre une escalade de tensions sous une forme linéaire. Une autre ligne est visible à l'ouest des États baltes sur l'axe de passage entre les bases navales de Baltiisk et Kronstadt. Elle concentre les incidents entre OTAN et Russie que représente la figure 3.

Figure 3. Incidents impliquant l'OTAN et la Russie

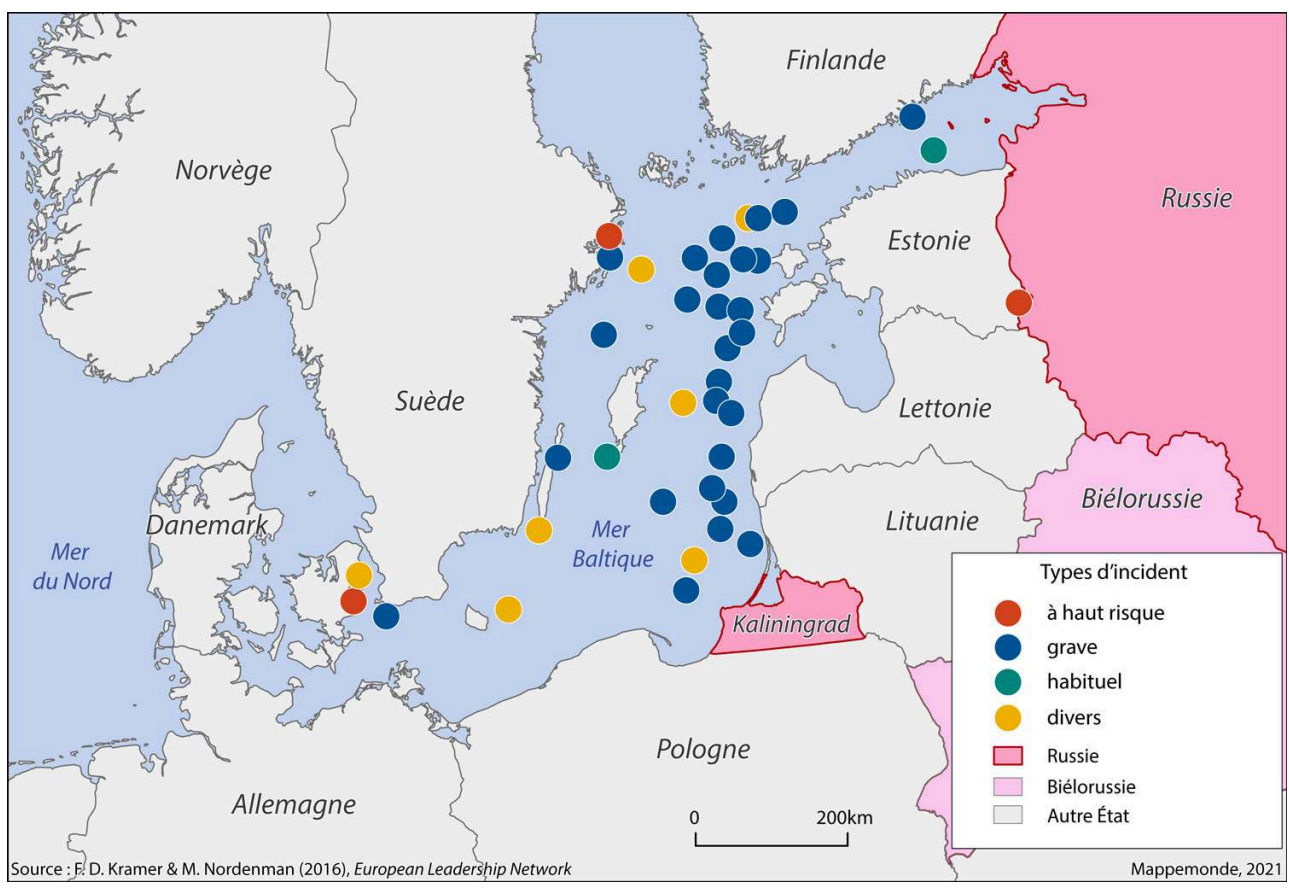

La lisière est souvent recherchée dans une perspective de fragilisation territoriale. Des différends territoriaux subsistent en mer Baltique et ont été réactivés par le choix d'un tracé pour le gazoduc Nord Stream 1 (zone contestée entre le Danemark et la Pologne). D'autre part, les rumeurs sur l'incursion présumée d'avions, de navires (y compris de pêche) et de sous-marins russes dans les eaux territoriales nordiques ou baltes sont anciennes, régulières, et s'inscrivent dans une dénonciation de la violation des frontières maritimes. Plus couramment, il arrive que des bateaux ou des avions russes frôlent les limites maritimes de souveraineté sans forcément les dépasser. Côté russe, les mêmes accusations sont reprises dans plusieurs médias locaux et nationaux. L'image du chalutier rempli de matériel électronique et de systèmes de surveillance fait notamment l'objet d'une récupération symbolique dans chaque camp.

L'insularisation de territoires entiers est manifeste dans ce contexte. Le transit régulier de navires entre Kronstadt et Baltiisk engendre un encerclement à l'ouest des États baltes, tandis que des exercices militaires conjoints avec la Biélorussie, comme Zapad 2017, les encadrent sur leur partie orientale (figure 5). L'enclave de Kaliningrad crée une coupure sur le flanc méridional que seul le passage de Suwałki rend incomplète. La présence de missiles moyenne portée Iskander ajoute au sentiment de menace et d'enfermement. Côté européen et OTAN, les passages resserrés (choke points) et les corridors d'échanges sont largement surveillés, tout comme les frontières de 
Kaliningrad. La majeure partie des bases navales se situent à proximité des détroits danois et de l'entrée du golfe de Finlande, tandis que deux groupements tactiques multinationaux de l'OTAN, Orzysz et Rukla, encadrent l'enclave russe (figure 4). La construction d'un corridor de sécurité nordique autour des îles de Bornholm, Gotland (remilitarisée) et Åland participe également d'un encerclement des positions russes.

La sanctuarisation est une figure défensive qui rappelle les espaces fermés et no man's land de la guerre froide. Kaliningrad, qui accueille la base militaire de Baltiisk, est une zone difficile d'accès, ce qui n'a pas été sans poser problème au moment de la Coupe du monde de football 2018 (figure 7). Depuis 2011, la Russie a modernisé les forces armées implantées dans les oblasts de Kaliningrad et de Leningrad suivant un principe de sanctuarisation agressive. Elle a déployé plusieurs systèmes de déni d'accès et d'interdiction de zone (anti-access/area-denial [A2AD]) sur sa frontière occidentale (ministère des Armées, 2019). En Suède, l'île de Gotland a, quant à elle, été remilitarisée, ce qui est une première depuis la suppression du dernier régiment en 2005, avec un dispositif cependant relativement léger. Teva Meyer, qui a cartographié les implantations militaires, évoque un bataillon de 301 soldats, 11 chars Stridsvagn, 15 véhicules de combat Strf 90, 3 véhicules à haute mobilité Bandvagn 309 et 1 char du génie Bgbv $120^{16}$.

La barrière est une forme de sanctuarisation linéaire qui a principalement été établie contre les mobilités des biens, à côté d'un durcissement des visas pour les personnes. Les sanctions économiques ouvertes et réciproques ont été doublées par des réprimandes plus indirectes. Suite aux sanctions touchant la Russie, un embargo sur la plupart des produits alimentaires venant de l'Union européenne a été décrété par le Kremlin dès le 6 août 2014. Le 4 juin 2015, la Russie a imposé un nouvel embargo sur les poissons ou produits de la mer importés d'Estonie et de Lettonie, alors que près de la moitié de la production lettone se dirige vers le marché russe. Cette décision faisait suite à la découverte annoncée d'un taux excessif de benzopyrène, une substance cancérigène, dans les sprats lettons par le Service fédéral russe pour la Surveillance vétérinaire et phytosanitaire (Rosselkhoznadzor). Les produits issus de Russie ont également subi un embargo côté européen. Des stratégies de contournement ont cependant été établies notamment via la Biélorussie. 
Figure 4. Les discontinuités du lac baltique

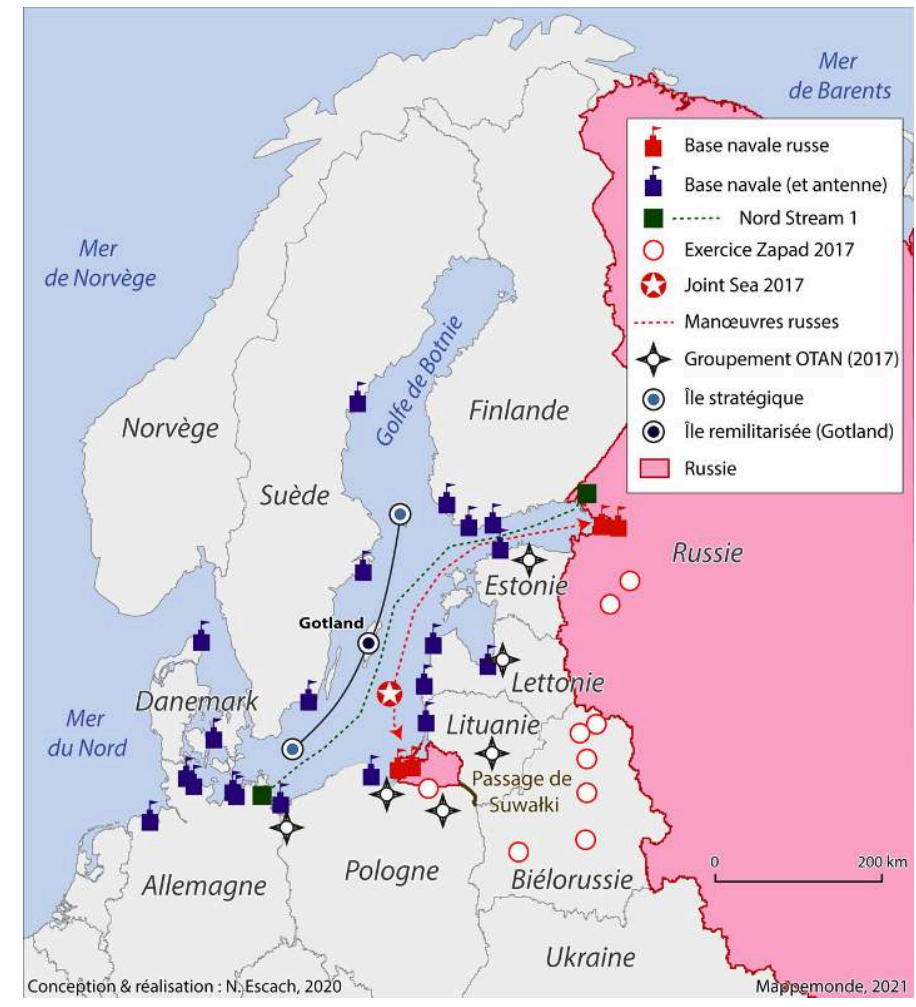

Figure 5. L'insularisation des États baltes

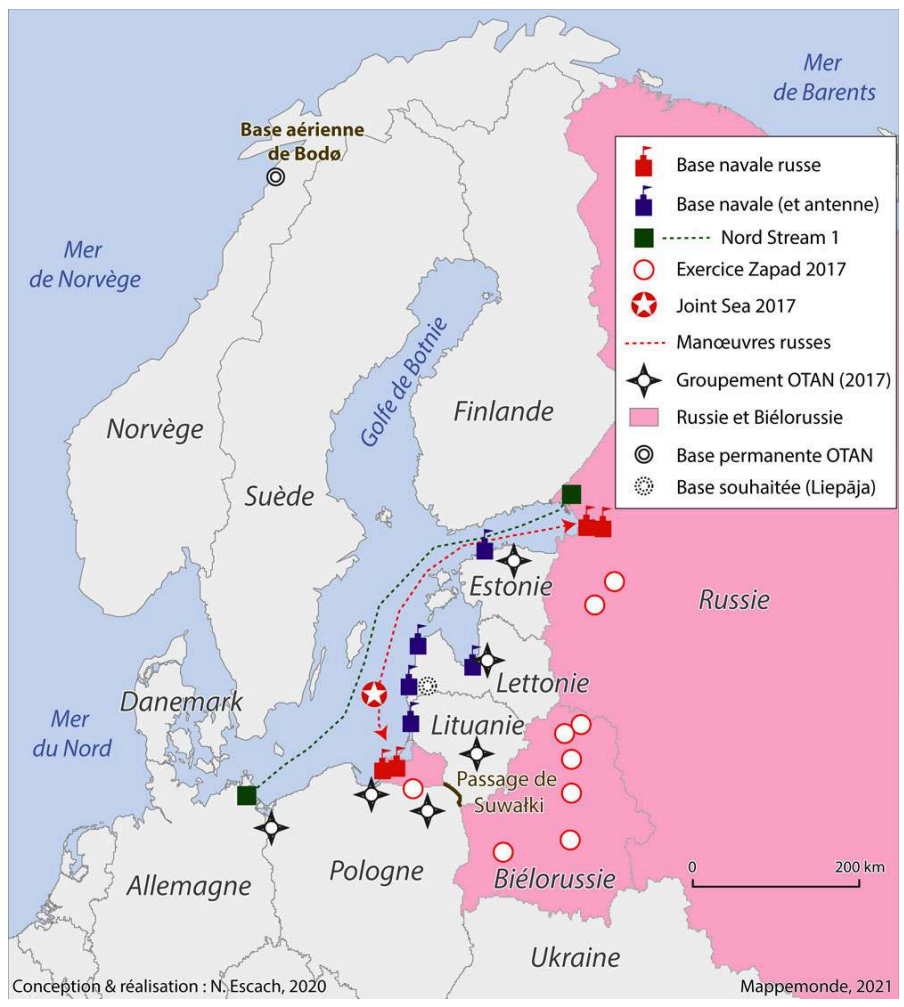


Figure 6. La tripartition de la mer Baltique

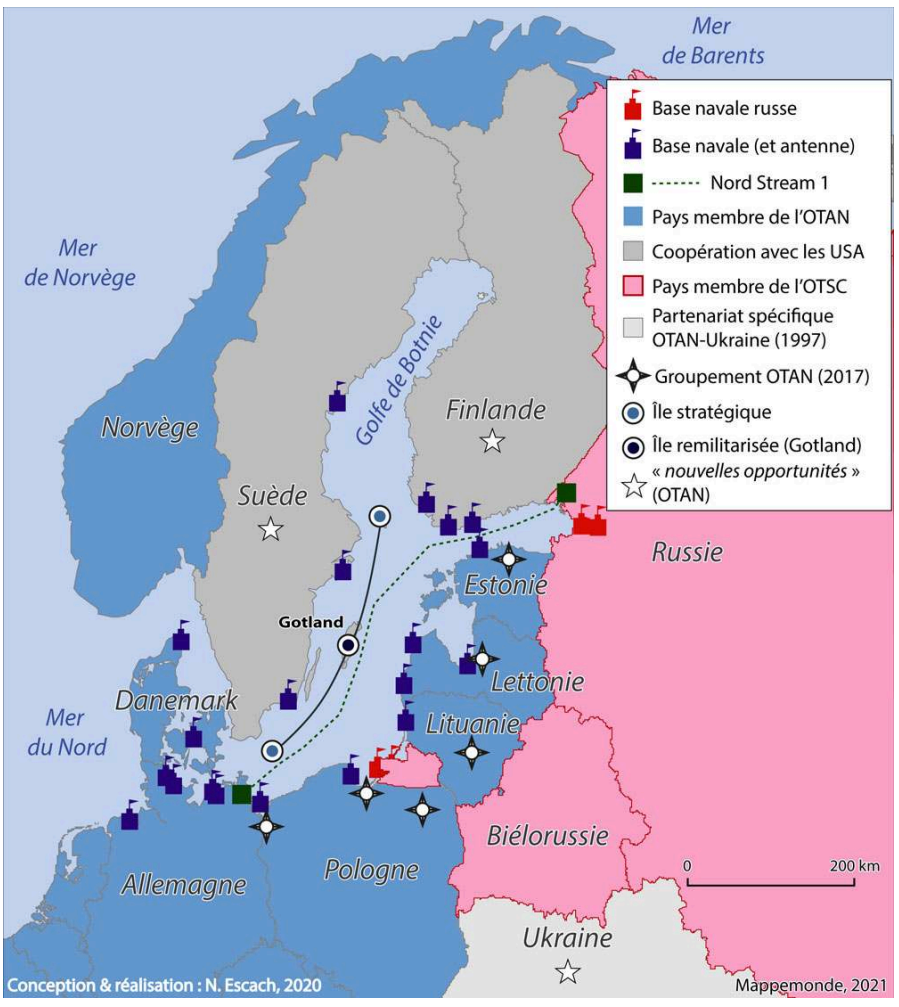

Figure 7. L'archipel russe en mer Baltique

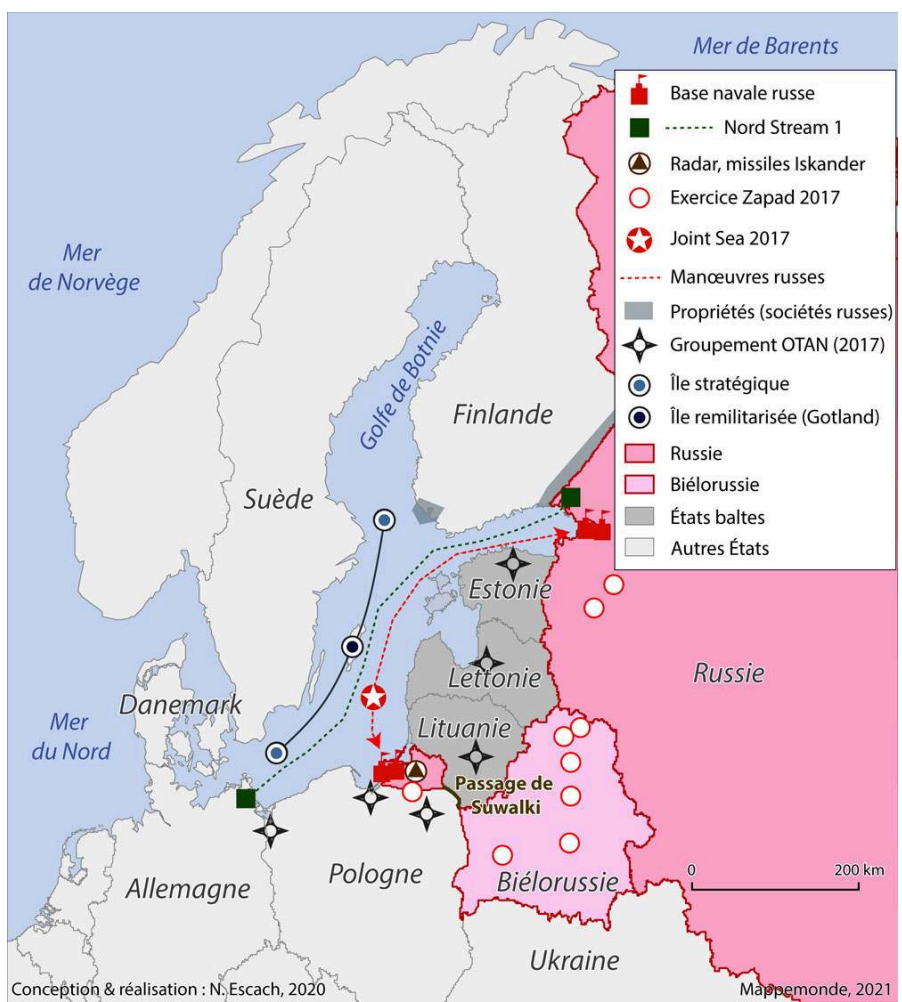

En mer Baltique, trois lignes divisent progressivement le lac baltique en cordons parallèles : une ligne de sécurité formée par l'île stratégique de Bornholm (Danemark), 
l'archipel d'Åland (Finlande) et l'île remilitarisée de Gotland (Suède), une ligne polémogène entre Kaliningrad et le golfe de Finlande aux voisinages des frontières maritimes baltes et une dernière ligne de tension autour de la frontière orientale de l'Union européenne (voir : https://halshs.archives-ouvertes.fr/halshs-03142250).

Face à ce regain de conflictualité, les réponses apportées ont été de plusieurs ordres (figure 7). À un niveau transnational, transfrontalier et international, plusieurs actions ont été engagées : une surveillance renforcée des frontières et la construction de grilles et de clôtures, des missions dans le cadre de l'OTAN à l'image de l'Arctic Challenge 2017 (ACE 17) en Norvège, Suède et Finlande, la participation aux contingents des groupements tactiques multinationaux de l'OTAN, une coopération renforcée avec les États-Unis. À un niveau national, les décisions prises ont été sans précédent: une augmentation du budget de la défense (Suède, Lituanie, Lettonie, Danemark, Norvège), un rétablissement $\mathrm{du}$ service militaire (Suède et Lituanie), la publication très médiatisée de livrets afin de préparer les habitants à une hypothétique invasion russe (Suède, Lituanie). À un niveau local, plusieurs initiatives ont contribué à construire " une territorialisation de la menace » (celle-ci s'incarnant dans un système spatial de protection reflétant le niveau d'anxiété ressenti ou supposé) : remilitarisation de l'île de Gotland, apparition de milices paramilitaires anti-Russes dans les États baltes, préemption foncière ou interdiction d'acquisitions étrangères à proximité de lieux stratégiques, réhabilitation de centaines de kilomètres de tunnels et de couloirs des années 1960 à Helsinki pour y établir une «ville souterraine » en cas d'invasion (400 locaux d'un volume de 9 millions de mètres cubes vont être établis en sous-sol d'ici à 2020 , permettant à la fois une protection de la population et un gain de densité dans le tissu urbain), reconversion de friches dans les États baltes pouvant accueillir de futures implantations militaires.

Figure 8. Les interconnexions fonctionnelles entre la Russie et la Baltique orientale

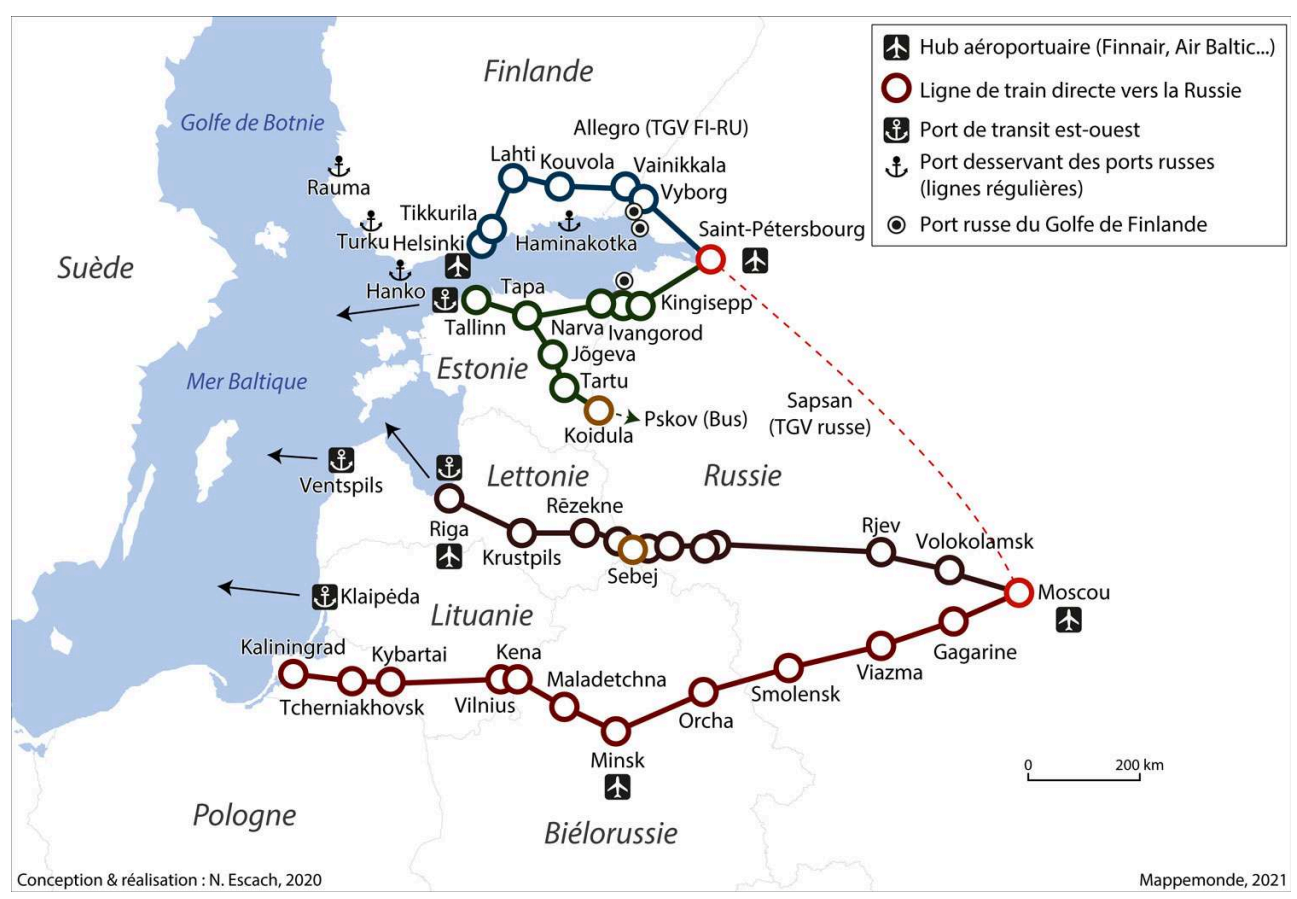




\section{De la fragilité à la vulnérabilité des territoires baltiques}

Les tensions et les conflits hybrides en mer Baltique ont généré un renforcement des vulnérabilités affectant directement les coopérations territoriales.

La vulnérabilité a avant tout été d'ordre spatial pour des territoires se sentant progressivement "circonscrits ", à l'image des États baltes entourés par la Russie (sur terre et en mer). L'effet de proximité joue un rôle substantiel, en raison de l'utilisation par les Russes d'armes médiatiques et cyber auprès des populations russophones des régions orientales de l'Union européenne. Comme le disait Milan Kundera, « un petit État peut disparaître, et il le sait ». Estonie, Lettonie et Lituanie ne savent plus s'ils peuvent compter sur les États-Unis de Donald Trump ${ }^{17}$, mais ne croient pas forcément, à l'heure actuelle, à une protection militaire des États de l'Union européenne. Les pays nordiques sont, de leur côté, impliqués dans deux foyers de tensions qui se font écho: la mer Baltique et l'océan Arctique dans lequel certains ont, sur le papier, des intérêts majeurs (Norvège, Danemark via le Groenland). Dans un contexte tendu, la région baltique est rarement considérée comme l'échelle d'action la plus efficace. James Norman Mattis, secrétaire américain à la Défense, s'est, par exemple, rendu en novembre 2017 à Helsinki pour un sommet du groupe du Nord avant de signer, en mai 2018, un accord de coopération militaire avec les ministres suédois et finlandais de la Défense.

La vulnérabilité spatiale est d'autant plus importante que l'organisation même de l'espace baltique est encore largement structurée par l'axe est-ouest et par des supports techniques hérités (écartement russe des voies ferroviaires). La persistance des territoires et l'inertie spatiale sont très fortes, comme le montrent les liens fonctionnels entre la Baltique orientale et la Russie représentés sur la figure 8. La construction au cours de la décennie 2000 de ports nationaux russes dans le golfe de Finlande a un peu plus fragilisé les ports de transit baltes (Tallinn, Riga, Ventspils, Klaipéda) qui ont dû diversifier leur hinterland. Ces interdépendances sont d'ailleurs instrumentalisées des deux côtés. Suite à l'instauration, en 2013, par la Russie, d'un régime de contrôle douanier renforcé à la frontière et de l'interdiction d'importer des produits laitiers lituaniens, le ministre des Affaires étrangères lituanien, Linas Linkevičius, a déclaré : "Comme vous le savez, la région de Kaliningrad est isolée, géographiquement isolée, ce qui nous permettrait de prendre des mesures pour interrompre le trafic. Nous pourrions intercepter les trains, mais aussi l'approvisionnement en marchandises. C'est théoriquement possible ${ }^{18}$. 
Figure 9. Les municipalités connectées à des partenaires russes par les projets INTERREG V-B

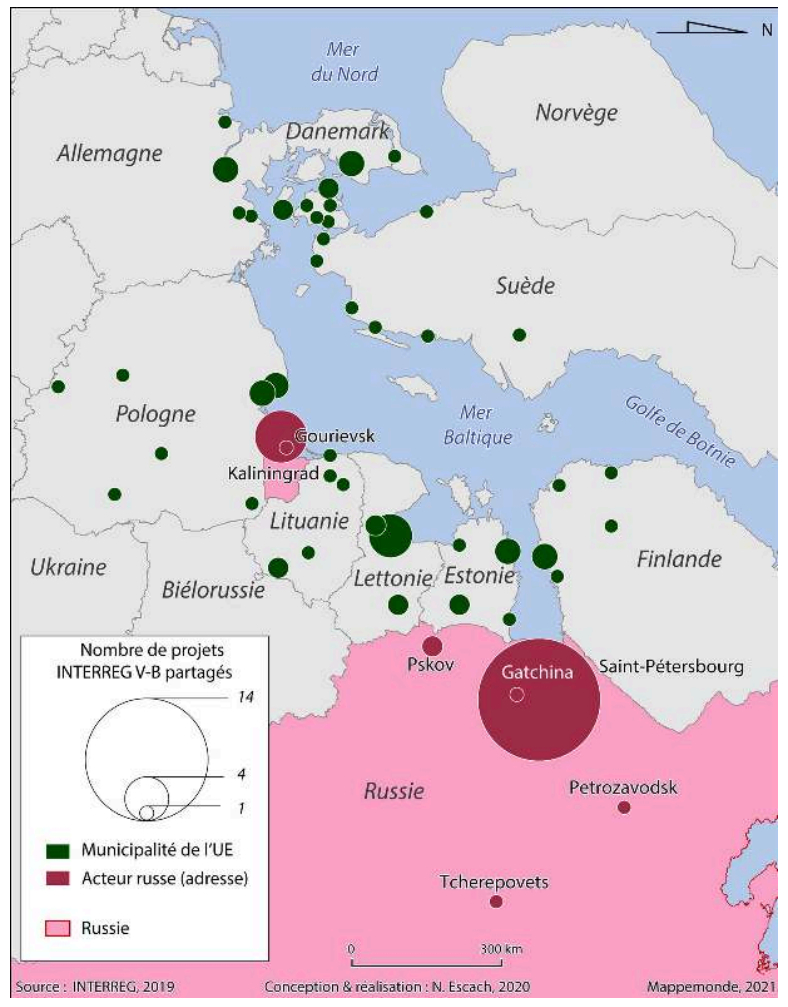

27 La vulnérabilité spatiale peut devenir interterritoriale lorsque les liens entre acteurs de différents niveaux géographiques ne peuvent plus s'exercer normalement. Les jumelages entre municipalités nordiques, baltes et russes sont relativement nombreux, avec des portes d'entrée privilégiées comme Saint-Pétersbourg, Kaliningrad, Kingisepp ou Petrozavodsk. L'Union des villes de la Baltique (UBC) met régulièrement à jour un document listant ces coopérations que nous avons reproduit dans cet article (tableau 1).

Tableau 1. Les villes de l'Union des villes de la Baltique jumelées avec des municipalités russes

\begin{tabular}{|l|l|}
\hline Villes de l'Union des villes de la Baltique & Villes russes jumelées \\
\hline Aarhus & Saint-Pétersbourg \\
\hline Elbląg & Baltiisk, Kaliningrad \\
\hline Espoo & Gatchina, Sotchi \\
\hline Gdansk & Kaliningrad, Saint-Pétersbourg \\
\hline Gdynia & Kaliningrad \\
\hline Jelgava & Magadan \\
\hline Jõhvi & Kingisepp \\
\hline Jyväskylä & Iaroslavl \\
\hline
\end{tabular}




\begin{tabular}{|l|l|}
\hline Kalmar & Kaliningrad \\
\hline Kaunas & Kaliningrad \\
\hline Klaipėda & Tcherepovets, Kaliningrad \\
\hline Kotka & Kronstadt \\
\hline Narva & $\begin{array}{l}\text { Ivangorod, } \\
\text { Kingisepp } \\
\text { Petrozavodsk } \\
\text { Kirov District (Saint-Pétersbourg), } \\
\text { Yugo-Zapadny Administrative District (Moscou) }\end{array}$ \\
\hline Örebro & Novgorod \\
\hline Riga & Moscou, Saint-Pétersbourg \\
\hline Sillamäe & Kingisepp \\
\hline Taurage & Sovetsk \\
\hline Turku & Saint-Pétersbourg \\
\hline Umeå & Petchory \\
\hline Võruaravodsk \\
\hline
\end{tabular}

Source : UBC, 2019

Pourtant, dans plusieurs pays riverains, le nombre des jumelages avec la Russie a globalement diminué sur la période 2007-2019. La base de données de l'Association suédoise des collectivités territoriales (Sveriges Kommuner och Landsting, SKL) est une source précieuse d'information étayant cette dynamique. En 2007, 35 jumelages étaient recensés entre villes suédoises et russes. En 2011, ils ne sont plus que 27, pour tomber à 16 en 2019. Beaucoup de ces liens sont d'ailleurs en sommeil. Certaines villes suédoises comme Sorsele ont pu recevoir des fonds SIDA (Agence suédoise de développement et coopération internationale) pour mener à bien leurs échanges. Les relations les plus actives se situent, de fait, dans la partie septentrionale du pays avec des cas de coopérations fructueuses, notamment celles de Luleå avec la ville de Mourmansk dans les champs de la culture, du tourisme et de l'économie.

La cartographie des liens entre acteurs, au sein des projets transnationaux INTERREG V-B (2014-2020), révèle une présence notable d'acteurs russes, principalement des universités et des centres de recherche, en comparaison avec la période 2007-2013 (figures 9 et 10). 42 projets INTERREG V-B sur 145, listés en 2019, associent formellement des partenaires russes aux profils variés. 14 de ces projets seulement sont labellisés projets pilotes (flagship projects) pour la macro-région baltique. Cependant, la lecture des cartes, que les municipalités seules ou l'ensemble des acteurs locaux soient pris en compte côté Union européenne et Norvège (les acteurs russes sont, eux, 
considérés dans leur globalité), atteste un net différentiel d'implication. Face à un ensemble conséquent de villes concernées dans les pays nordiques, baltes, en Allemagne et en Pologne, la Russie est engagée à travers seulement quelques portes d'entrée identifiables, qui correspondent aux villes déjà connectées via des jumelages : Saint-Pétersbourg en tête, qui conserve ainsi son rôle de "fenêtre vers l'Occident " (statut historique qui avait justifié sa fondation par Pierre le Grand), suivie de Kaliningrad, Pskov, puis Petrozavodsk, Tcherepovets, Arkhangelsk et Gouryevsk.

Figure 10. Les acteurs locaux connectés à des partenaires russes par des projets INTERREG IV-B

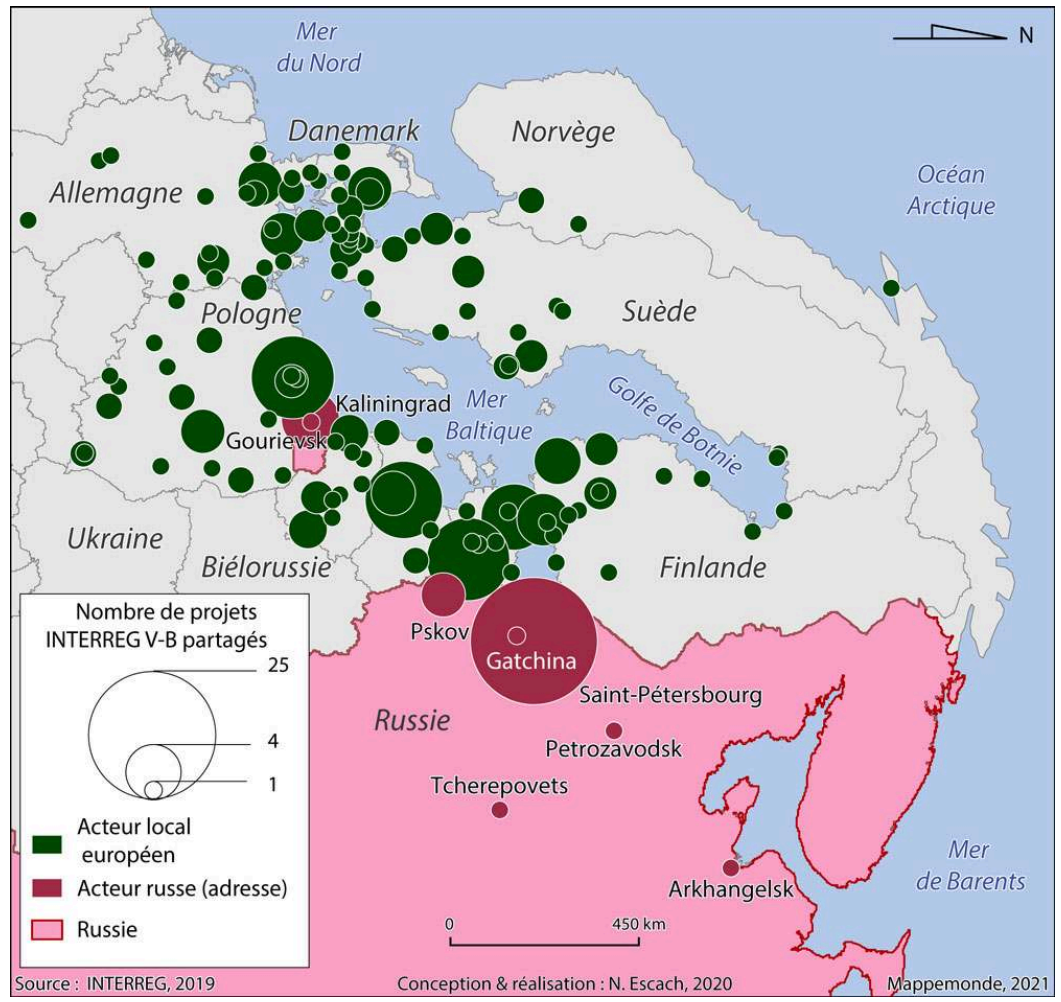

Acteurs locaux : universités, centres de recherche, municipalités, acteurs municipaux, ports, associations et ONG.

Cette «interterritorialité contrariée» a été renforcée par une vulnérabilité institutionnelle et financière en raison de sanctions économiques et de la nonsignature d'accords de cofinancement remettant en cause des projets transfrontaliers ou transnationaux. Un retard, certes habituel, dans la signature des accords financiers de coopération entre la Commission européenne, la Fédération de Russie et l'Allemagne n'a pas permis aux vingt-quatre partenaires russes d'obtenir une avance de paiement sur les projets entamés dans le cadre des programmes transnationaux INTERREG. Les sanctions économiques imposées par l'Union européenne à la Russie ont par ailleurs bloqué les actions du Partenariat environnemental de la Dimension septentrionale et les contributions financières de la Banque européenne d'investissement, de la Banque européenne pour la reconstruction et le développement, de la Banque nordique d'investissement et de la Société nordique de financement pour l'environnement, sur plusieurs volets écologiques.

31 Le manque de prévisibilité financière a donc engendré une vulnérabilité environnementale. Les deux partenaires russes du projet transnational INTERREG BEST 
(2017-2020), associant des acteurs finlandais, polonais, baltes et russes autour du traitement des eaux usées de l'industrie, devaient recevoir $50 \%$ du budget de la Fédération de Russie et $50 \%$ de l'Instrument européen de voisinage et de partenariat (ENI). Après seize mois de projet, ils n'ont reçu que $30 \%$ du montant prévu, en avance, en raison du retard de signature de l'accord-cadre financier, puis du délai d'un an pris par les autorités russes pour virer la somme sur les comptes européens. Alors que les travaux collectifs ont débuté le $1^{\mathrm{er}}$ octobre 2017, le versement n'a eu lieu qu'en janvier 2019. Devant une si longue incertitude l'un des deux partenaires russes (de Gatchina) a dû entre temps quitter le projet, l'autre rattrape depuis péniblement son retard sur les workpackages. Tous les investissements prévus n'ont pas pu être menés, les tâches ont été réorganisées (notamment le rétroplanning), et le leader du projet, la ville d'Helsinki, a dû trouver des solutions avec le secrétariat et l'autorité de gestion pour couvrir les frais de transport vers les réunions collectives ${ }^{19}$. L'abandon des investissements pour la mesure des eaux usées industrielles se jetant dans la rivière Izhora est la conséquence directe de l'ajustement du projet. Sur la liste des partenaires de l'ensemble des autres projets INTERREG V-B de nombreuses mentions « retrait " sont d'ailleurs signalées. Parallèlement, dans l'oblast de Leningrad, 184 sites ne disposent pas de stations d'épuration et de traitement des eaux usées performantes. Les abords des réacteurs nucléaires de Sosnovy Bor (près de Saint-Pétersbourg) cumulent, par exemple, une fuite de substances dangereuses et un non-traitement des eaux, ce qui pourrait avoir d'importantes conséquences sur les régions limitrophes de l'UE si le dialogue avec la Russie sur ce point n'est pas rétabli. Le rapport réalisé par Christina Gestrin, ancienne députée et observatrice auprès d'Helcom, est assez éloquent sur le sujet (Gestrin, 2018). Certes, les tensions hybrides ne sont pas les seules responsables d'une difficulté ancienne à mener des projets conjoints entre les acteurs de l'UE et de la Russie, mais leur multiplication a pu accentuer les obstacles à une conduite sereine des coopérations ${ }^{20}$.

Face à des vulnérabilités de diverses natures, la réponse des acteurs baltiques est très peu régionale. Les tensions ont même réactivé des visions stratégiques particulièrement différenciées. Les États baltes et la Finlande continuent de regarder vers l'Asie sur le plan du tourisme, du commerce et des transports. Ils pourraient également être concernés par une nouvelle route sud/nord vers l'Arctique (l'Arctic Corridor se prolongeant vers la Via baltica et le Rail baltica). La Norvège mène, quant à elle, une politique active pour la sécurisation de l'Arctique. Le Danemark s'appuie en revanche très peu sur ses atouts au Groenland et, dans une moindre mesure, dans les îles Féroé. La Pologne est engagée dans un projet de connexion sur la route des "Varègues aux Grecs ». Elle défend son rôle central au sein de "l'Initiative des Trois Mers » (Baltique, Adriatique, mer Noire), héritage de l'Intermarium de Józef Piłsudski. La Suède constitue, en ce sens, un pivot attentif à l'escalade des tensions en mer Baltique avec la remilitarisation de Gotland comme avant-poste.

\section{Conclusion}

Le géopoliticien suédois Gunnar Åselius se demandait « s'il faut considérer la Baltique comme une mer intérieure nord-européenne bien délimitée ou comme un golfe de l'Atlantique-est» (Åselius, 2001, p. 83). Aujourd'hui les conflits hybrides en mer Baltique dépassent largement son cadre géographique. Des rivalités de diverses natures 
(tensions, conflits ouverts, guerres) rassemblent les mêmes protagonistes (Russie, Chine, UE, OTAN) sur plusieurs théâtres (Asie centrale et Caucase, Baltique, Arctique). Dans ces oppositions se noue une définition des nouvelles routes est-ouest entre l'Europe, qui peine à se réinventer, et l'Asie qui s'impose démographiquement et économiquement. La Russie affirme ses ambitions eurasiatiques, tandis que la Chine cherche à étendre ses nouvelles routes de la Soie (terrestres et maritimes) en investissant de plus en plus massivement dans la région. Le tunnel prévu entre Tallinn et Helsinki a ainsi été controversé en raison d'un montage financier qui pourrait laisser la part belle à des fonds chinois (avec une participation à hauteur de 15 milliards d'euros de Touchstone Capital Partners). La coopération baltique subit le même risque de délitement que les pays européens face à ces nouveaux partenariats.

Où la coopération baltique est-elle passée ? Les organisations régionales investissent, de fait, trop peu les questions de sécurité dure, bien que le réseau NB8 ${ }^{21}$ (Nordic-Baltic 8) aurait un rôle certain à jouer. Le UK Joint Expeditionary Force (JEF), qui rassemble la Grande-Bretagne, la Suède, le Danemark, la Finlande, l'Estonie, la Lettonie, la Lituanie, les Pays-Bas et la Norvège, pourrait également constituer un forum crédible, bien que la Pologne n'en fasse pas partie. Ce manque de gouvernance à un niveau transnational s'ajoute au Brexit et à une méfiance de la part des Baltes envers la capacité de l'Union européenne à assurer leur sécurité. Lors de la Conférence de Munich sur la Paix et la Sécurité de 2018, la présidente estonienne Kersti Kaljulaid ne s'était pas privée de rappeler à certains membres de l'OTAN l'engagement non tenu d'allouer une part plus substantielle de leur budget à la défense. L'Allemagne était visée : son budget de la défense représentera en effet $1,5 \%$ du produit intérieur brut (PIB) du pays en 2021, contre $1,2 \%$ aujourd'hui, loin de l'objectif de $2 \%$ fixé par l'OTAN à ses membres d'ici à 2024.

La vulnérabilité visible à une échelle locale touche également l'échelle transnationale. Après avoir baigné dans un discours enjôleur sur le retour du lac baltique au début de la décennie 1990, les acteurs régionaux dessinent aujourd'hui des stratégies de plus en plus différenciées, au besoin bilatérales (à l'image des Suédois et des Finlandais traitant directement avec les États-Unis), ce qui risque d'entacher la réactivité face aux nœuds géopolitiques, mais aussi la nécessaire proactivité dans des forums politiques transnationaux ou transfrontaliers autour de sujets essentiels comme l'environnement. L'état de la mer Baltique ne pourra s'améliorer sans un dialogue sécuritaire accru entre les différents États riverains sans exception.

\section{BIBLIOGRAPHIE}

ÅSELIUS G. (2001). « La géographie militaire suédoise et scandinave dans une perspective historique ». Stratégique, $\mathrm{n}^{\circ}$ 81, p. 77-103.

BAYOu C. (2015). « Sécurité. Avis de tempête sur la Baltique ». P@ges Europe, 15 juillet 2015, La

Documentation française. 
BELLIGoli S. (2012). « North Stream à l'épreuve des relations euro-russes ». IEE-Document, $\mathrm{n}^{\circ} 47,76$ p.

BRUNET R. (1980). « La composition des modèles dans l'analyse spatiale ». Espace géographique, t. 9, $\mathrm{n}^{\circ} 4$, p. 253-265.

BRUNET R. (1986). « La carte-modèle et les chorèmes ». Mappemonde, $\mathrm{n}^{\circ}$ 4, p. 2-6. En ligne : http:// www.mgm.fr/PUB/Mappemonde/Mappe486R.html

CHALVIn, A. (2007). « L'ombre du soldat de bronze ». Le Courrier des pays de l'Est, vol. 4, n 1062, p. 6-16.

CHILLAUD M. (2007). La démarche stratégique des États baltes dans l'architecture européenne de sécurité et de défense. Une politique fondée sur une dialectique identitaire et militaire. De la restauration de leur indépendance aux commémorations russes du soixantième anniversaire de la victoire contre l'Allemagne nazie. Thèse soutenue le 30 mars 2007 sous la direction de Nathalie Blanc-Noël, 615 p.

ESCACH N. (2014). Réseaux de villes et recompositions interterritoriales dans l'espace baltique. Thèse soutenue le 14 novembre 2014 sous la direction de Lydia Coudroy de Lille et Boris Grésillon, 463 p. ESCACH N. (2016). «Partage de la mer et nouveaux conflits géopolitiques en Baltique ». Hérodote, $\mathrm{n}^{\circ} 163$, p. 175-191.

GESTRIN C. (2018). Environmental Co-operation in the Baltic Sea Region. Report of the Nordic Council, 8 p.

HASANEN M. (2017). Russian Real Estate Purchases in Finland, 1990-2016. Mémoire de master soutenu à l'université d'État du Dakota du Sud, 65 p. En ligne : https://openprairie.sdstate.edu/cgi/ viewcontent.cgi?article $=3191 \&$ context $=$ etd

LARSSON R. (2007). “Nord Stream, Sweden and Baltic Sea Security”. FOI. Base Data Report. Defense Analysis, $110 \mathrm{p}$.

MARIN A. (2009). «Argument Baltique : faux prétexte et modèles justes ». Outre Terre, $\mathrm{n}^{\circ} 23$, p. 347-362.

MURPHY M., HOFFMAN F. G., SCHAUB G. Jr (2016). Hybrid Maritime Warfare and the Baltic Sea Region. Academic report. Centre for military studies. Université de Copenhague, $62 \mathrm{p}$.

MINISTÈRE DES ARMÉES. (2019). « La France face aux enjeux de sécurité en Baltique ». Plaquette DGRIS, $12 \mathrm{p}$.

RADIN A. (2017). Hybrid Warfare in the BalticsThreats and Potential Responses. Santa Monica : RAND Corporation, $58 \mathrm{p}$.

RUBEN E. (2014). La ligne des glaces. Paris : Rivages, $280 \mathrm{p}$.

TÉTART F. (2007). Géopolitique de Kaliningrad: une «île » russe au sein de l'Union européenne élargie. Paris : Presses universitaires de Paris-Sorbonne, $478 \mathrm{p}$.

\section{NOTES}

1. Avec des travaux s'intéressant à l'unité de la région sous un angle géohistorique associant, par exemple, Matti Männikkö et Klaus Zernack, puis David Kirby et Matti Klinge, avant que des politistes ne participent au lancement des premiers réseaux d'acteurs, à la fin des années 1980 et au début des années 1990, en entretenant malgré eux une confusion parfois gênante entre lignes de programmation politique et lignes d'analyse scientifique. 
2. Cette seconde période débute au début des années 1990 avec les travaux de chercheurs étrangers post-structuralistes comme Iver B. Neumann et Ole Wæver (region building approach). Ce dernier déclare même en 1992 : "Does the Baltic Sea Region exist? No, but it will". En France, la prise de conscience est bien plus tardive et s'inspire nettement de ces publications ainsi que de chercheurs allemands.

3. De manière paradoxale, ce retour des conflits dans les recherches sur l'espace baltique intervient peu après l'élargissement oriental de l'Union européenne (2004), ce qui n'a pas empêché, au moment du lancement de la macro-région baltique (2009), la publication de nombreuses contributions sur les outils mobilisés par les autorités européennes pour renforcer la coopération des États riverains autour de défis communs (suscitant des débats sur les logiques institutionnelles adoptées).

4. Avec pour les États baltes, une attention portée aux minorités.

5. Dans un sens littéral, un système spatial vulnérable n'a plus les capacités de résilience suffisantes pour ne pas être désorganisé en cas de choc. Il est exposé et fragile car son "arsenal de protection » n'est pas assez solide. Il n'est pas/plus robuste.

6. L'adjectif insidieux traduit parfaitement une accumulation de tensions qui se répandent sournoisement et dont l'apparente bénignité peut masquer de lourdes conséquences.

7. Marin A. (2009). « Faux prétexte et modèles justes ». Outre Terre, $\mathrm{n}^{\circ} 23$, p. 349.

8. Conférence de Vytautas Isoda, directeur du département des sciences politiques à l'Académie militaire de Lituanie, colloque Security in the Baltic Sea Region, IRSEM, octobre 2019.

9. The Estonian Foreign Intelligence Service. (2019). International Security and Estonia, $72 \mathrm{p}$.

10. Émission «De Vilnius à Tallinn: contenir la Russie», Cultures Monde, France Culture, $07 / 02 / 2020$.

11. Des intrusions présagées de sous-marins russes dans des eaux territoriales d'autres pays riverains ont été relayées régulièrement pendant la guerre froide.

12. Voir https://nemrod-ecds.com/?p=3186 (consulté le 24/03/2019).

13. National Land Survey of Finland.

14. Voir https://yle.fi/uutiset/3-8664889 (consulté le 24/03/2019). - Uudempia hybridisodan ilmiöitä ovat pakolaisvirrat, informaatiosota ja maanhankinta strategisten kohteiden läheltä, listaa komitean sihteeristön yleissihteeri Tatu Mikkola.

15. http://www.defens-aero.com/2015/06/arctic-challenge-exercise-2015-un-red-flageuropeen.html, consulté le 29/03/2019.

16. https://geoposvea.hypotheses.org/512, consulté le 29/03/2019.

17. L'article a été rédigé avant l'investiture de Joe Biden le 20 janvier 2021.

18. «Lithuania warns Russia over pressuring its neighbors ", Reuters, 2 octobre 2013.

19. Les frais de transport sont souvent, en sus de difficultés pour obtenir les autorisations administratives, un obstacle pour la participation de partenaires russes ou biélorusses aux projets INTERREG.

20. "Eco-cooperation with Russia vital for Baltics despite sanctions", EUobserver, 6 mars 2018.

21. NB8 est une plateforme de coopération rassemblant l'Islande, la Norvège, le Danemark, la Suède, la Finlande, l'Estonie, la Lettonie et la Lituanie. Elle permet des réunions régulières principalement entre premiers ministres et ministres des Affaires étrangères mais aussi entre ministres, secrétaires d'État, parlementaires et experts sur une variété importante de thématiques (sécurité, surveillance financière, éducation, culture). 


\section{RÉSUMÉS}

Depuis le milieu de la décennie 2000, les questions de sécurité dure, évacuées après la chute du Rideau de fer, ont rejailli progressivement entre partenaires baltiques. La crise de 2014 en Crimée a attisé les craintes quant à la menace russe. Contrairement au cas ukrainien, le front baltique est pour l'instant condamné à rester immobile, ce qui ouvre tout grand la possibilité de conflits hybrides chahutant la limite plus qu'ils ne la déplacent. Bien que de plus en plus médiatisée, la zone baltique est devenue le décor de dislocations légères, de conflits larvés, d'intimidations sourdes, en somme d'une géopolitique insidieuse. Ces perturbations en mode mineur, produits de tensions faibles, n'ont de force que leur accumulation, laquelle rend structurants et dangereux des événements diffus, discontinus et de basse intensité. La multiplication des fragilisations a accentué la vulnérabilité des territoires à toutes les échelles menaçant ou altérant l'interterritorialité si souvent associée à l'espace baltique.

Since the mid 2000s, security issues, which had been evacuated after the fall of the Iron Curtain, have gradually resurfaced between Baltic partners. The 2014 Crimean crisis fueled fears about the Russian threat. Unlike Ukraine, the Baltic front is momentarily immobilised, which opens the horizon wide to hybrid conflicts that challenges without moving the limits. Although increasingly publicized, the Baltic area has become the theater of slight dislocations, latent conflicts, muffled intimidation, in short, insidious geopolitics. What force these minor disturbances, the product of slight tensions, have is only in their accumulation. It is this accretion that structures and renders dangerous the dispersed, halting events of low intensity. The multiplication of fractures has accentuated territorial vulnerabilities at all levels, threatening and even altering the inter-territoriality so often associated with the Baltic area.

Desde mediados de la década de 2000 las cuestiones de seguridad, olvidadas después de la caída del Telón de Acero, han resurgido gradualmente entre los socios bálticos con la crisis de Crimea de 2014, que acrecentó los temores a una amenaza potencial rusa. A diferencia del caso ucraniano, el frente báltico continua inmóvil aunque existe la posibilidad de conflictos híbridos que alteren los límites fronterizos. En un contexto muy mediático, la zona báltica se ha convertido en un escenario geopolítico insidioso con pequeñas dislocaciones, conflictos latentes o intimidaciones amortiguada. Estas perturbaciones menores, producto de bajas tensiones, sólo tendrán fuerza en su acumulación, pudiendo convertir situaciones difusas, discontinuas y de baja intensidad en otras estructurantes y peligrosas. Se está alterando la interterritorialidad del espacio báltico por la multiplicación de estas tensiones, que están acentuado su vulnerabilidad en todas sus escalas.

\section{INDEX}

Thèmes : La Baltique. Un espace d'intégration mais aussi de concurrence et de rivalités

Keywords : Baltic Sea, Hybrid Warfare, conflicts, geopolitics, regionalization

Mots-clés : Baltique, tensions hybrides, conflits, géopolitique, régionalisation

Palabras claves : Báltico, tensiones híbridas, conflictos, geopolítica, regionalización 